\title{
ESTIMATIVA DE PARÂMETROS FLORESTAIS EM ÁREA DE CERRADO A PARTIR DE IMAGENS DO SENSOR OLI LANDSAT 8
}

\author{
Micael Moreira Santos ${ }^{1 *}$, Igor Elói Silva Machado², Edmar Vinicius Carvalho ${ }^{3}$, \\ Marcelo Ribeiro Viola ${ }^{4}$, Marcos Giongo ${ }^{3}$

\begin{abstract}
${ }^{1 *}$ Universidade Federal do Paraná, Pós-Graduação em Engenharia Florestal, Curitiba, Paraná, Brasil - moreirasmicael@ gmail.com ${ }^{3}$ Universidade Federal do Tocantins, Centro de Monitoramento Ambiental e Manejo do Fogo, Gurupi, Tocantins, Brasil giongo@uft.edu.br; carvalho.ev@uft.edu.br
\end{abstract} \\ ${ }^{2}$ Universidade Federal do Tocantins, Pós-Graduação em Ciências Florestais e Ambientais, Gurupi, Tocantins, Brasil - igeloi@ hotmail.com \\ ${ }^{4}$ Universidade Federal de Lavras, Departamento de Engenharia, Lavras, Minas Gerais, Brasil - marcelo.viola@deg.ufla.br
}

Recebido para publicação: 05/08/2016 - Aceito para publicação: 19/12/2016

\begin{abstract}
Resumo
A obtenção de dados que caracterizam determinado fragmento florestal esbarra na dificuldade de processamento dos dados, nos altos custos demandados e no elevado tempo gasto com os inventários tradicionais. O uso de técnicas de Processamento Digital de Imagens (PDI), utilizando dados de sensoriamento remoto para estimar variáveis florestais, pode ser ferramenta útil e eficaz na complementação do inventário de campo. O presente trabalho teve como objetivo avaliar a viabilidade da utilização de imagens multiespectrais do sensor OLI Landsat 8, através de análise de regressão com dados de campo, para a estimativa de parâmetros florestais. Para isso foi realizado inventário com objetivo de obter os parâmetros florestais: Diâmetro à Altura do Peito (DAP); área basal $(\mathrm{G})$; altura total $(\mathrm{Ht})$; número de árvores $(\mathrm{N})$ e volume de madeira (V). Na etapa de PDI, foram utilizados os valores de reflectância e os índices de vegetação NDVI e SAVI nos pixels que abrangiam as parcelas do inventário, seguidos das análises de correlação entre as bandas e os parâmetros florestais. Em seguida foram feitos o ajuste dos modelos de regressão linear múltipla para a espacialização dos dados. Os melhores resultados, referentes aos valores de $\mathrm{R}^{2}{ }_{\text {aj }}$, para os modelos de DAP; G; $\mathrm{Ht} ; \mathrm{N}$ e V, foram de 0,57; 0,66;0,16;0,34 e 0,49, respectivamente. Os resultados permitem concluir sobre uso potencial da metodologia na estimação das variáveis DAP, G e V com uso do NDVI e das bandas referentes ao verde, vermelho e infravermelho médio.

Palavras-chave: Sensoriamento remoto; inventário florestal; reflectância; índice de vegetação.
\end{abstract}

\begin{abstract}
Estimation of forest parameters in Cerrado area from OLI Landsat 8 sensor. The obtaining of data that characterize certain forest fragment comes up against the difficult of data processing, high costs and demanded time spent with forest inventories. The use of image Digital Processing Techniques (DPT) with remote sensing data to estimate forest variables can be useful and effective tool to complement the field inventory. This study aimed to evaluate the viability of the utilization the multispectral images from OLI Landsat 8 sensor through regression analysis with field data for estimation of forest parameters. For this, it was done an inventory in order to get the forest parameters: diameter at breast height (DBH); basal area $(\mathrm{G})$; total height (Ht); number of trees (N) and timber volume (V). In DPT step, they were used the reflectance and the NDVI and SAVI vegetation index values from the pixels that cover the plots of the inventory and after it was conducted the correlation analysis among bands and forest parameters. Finally, they were made the adjustment of multiples linear regression models for spatialization data. The best results related to $\mathrm{R}^{2}{ }_{\mathrm{aj}}$ values for models of $\mathrm{DBH}$; $\mathrm{G}$; $\mathrm{Ht}$; $\mathrm{N}$; and $\mathrm{V}$, were $0.57 ; 0.66 ; 0.16 ; 0.34$ and 0.49 , respectively. The results were allowed to conclude about the use potential of the methodology for the estimation of the following traits DBH, G and V with using NDVI and the bands related the green, red and SWI.

Keywords: Remote sensing; forest inventory; reflectance; vegetation index.
\end{abstract}

\section{INTRODUÇÃO}

A estimativa de parâmetros florestais sempre foi alvo de estudos de novas técnicas que buscam facilitar a obtenção dos dados, o gerenciamento dos recursos florestais e diminuir os altos custos com inventários. No entanto, com a complexidade das informações para se otimizar o processo de tomada de decisão é comum observar esforços na busca de alternativas (CANAVESI et al., 2010).

Bolfe et al. (2004) ressaltam, ainda, que os métodos tradicionais de inventário florestal não são efetivos, pois geram demanda elevada no fluxo de informações, se deparando com a dificuldade em alocar, detalhar, organizar, interpretar e armazenar informações para formar um banco de dados confiável. Os inventários 
tradicionais, na maioria das situações, são caros e difíceis de serem realizados quando em grandes áreas ou quando as florestas se encontram em áreas remotas e de acesso dispendioso (ALVES et al., 2013).

Os avanços tecnológicos com relação aos dados de sensores remotos, nos últimos anos, e o desenvolvimento relacionado ao processamento de imagens, segundo Watzlawick et al. (2009), contribuem para melhor caracterização da estrutura da vegetação e, consequentemente, a quantificação de sua biomassa e estoque de carbono.

O processamento digital com imagens (PDI), obtidas de sensores remotos, tem como objetivo principal fornecer ferramentas que facilitem a visualização e a extração dos dados contidos na imagem. Tal procedimento, realizado com o auxílio de sistemas computacionais para análise e manipulação das imagens brutas (RIBEIRO et al., 2009), permite a obtenção de Índices de Vegetação (IVs) (HUETE, 1989) que possuem boa correlação com aspectos estruturais florestais (LEAL et al., 2013; CASTANHEIRA et al., 2014; ALMEIDA et al., 2015), sendo amplamente utilizados em trabalhos científicos.

Para Ferraz et al. (2014), as imagens dos sensores remotos, transformadas para IVs são de extrema importância e compõem ferramentas eficazes na estimação de variáveis florestais, como a produção de biomassa. Para obter os IVs, e eliminar a interferência atmosférica na imagem de satélite, faz-se necessário a transformação dos valores originais da imagem, composta por Números Digitais (ND), em valores de radiância/reflectância (GÜRTLER et al., 2005), em que diversos autores, como por exemplo, Viana et al. (2012), Leal et al. (2013) e Barros et al. (2015) usaram os valores de reflectância.

Tendo em vista a facilidade e disponibilidade de dados e sua ampla utilidade em trabalhos científicos que envolvem sensores remotos, tem-se como alternativa mais viável a exploração de dados dos sensores da série Landsat. Trabalhos como os de Lu et al. (2004), Berra et al. (2012) e Hentz et al. (2014) são alguns dos exemplos de utilização de imagens provenientes do satélite Landsat para a estimativa de variáveis de interesse florestal.

Parâmetros florestais como volume e biomassa são os mais encontrados em literatura como variáveis dependentes desejáveis a serem estimadas (WATZLAWICK et al., 2009; RIBEIRO et al., 2009; BERRA et al., 2012; LIMA JÚNIOR et al., 2014; BARROS et al., 2015). Isto pode ser feito por meio da correlação dos dados anteriormente amostrados em campo com as variáveis independentes, obtidas das imagens e dos IVs, e da obtenção de equações matemáticas que permitem espacializar as estimativas dos parâmetros de interesse.

$\mathrm{Lu}$ et al. (2004) relatam que, no decorrer dos últimos anos, muitas pesquisas baseadas em sensoriamento remoto focaram na estimativa de parâmetros florestais de povoamentos e em análises de correlação/regressão com o objetivo de descobrir o grau de relação entre a resposta espectral e os fatores estruturais da floresta. Os mesmos autores destacaram como variáveis ou parâmetros estruturais a área basal, biomassa, cobertura vegetal, Diâmetro a Altura do Peito (DAP), altura das árvores, densidade da vegetação e Índice de Área Foliar (IAF).

Considerando tal contexto, o objetivo deste trabalho foi avaliar a viabilidade da utilização de imagens multiespectrais do sensor OLI Landsat 8 através de análise de regressão com dados de campo, para a estimativa de parâmetros florestais em área de cerrado.

\section{MATERIAL E MÉTODOS}

O presente estudo foi realizado na região sul do estado do Tocantins, mais precisamente no município de Cariri do Tocantins, onde se encontra um remanescente de Cerrado com uma área de aproximadamente 506 hectares (ha). Dentro da área de estudo nota-se uma descontinuação da vegetação natural, com uma área de aproximadamente 39 ha, que pode ser observada na diferente tonalidade da imagem.

A área de estudo está inserida no sistema hidrográfico rio Araguaia (sub-bacia do rio Formoso) e no sistema hidrográfico rio Tocantins (sub-bacia do rio Santo Antônio). De acordo com a classificação climática de Thornthwaite o clima na região em estudo é do tipo C2wA'a', caracterizado como úmido subúmido e de moderada deficiência hídrica no inverno. A evapotranspiração potencial média anual é de aproximadamente $1.500 \mathrm{~mm}$, distribuida no verão em torno de $420 \mathrm{~mm}$, ao longo dos três meses consecutivos com temperatura mais elevadas (SEPLAN, 2012). Ao sul do remanescente, contornando a área se encontra o córrego Bacabal conforme dados da Seplan (2012), onde se tem vegetação de mata de galeria em grande parte de sua extensão. Tal vegetação é caracterizada pelos finos troncos das árvores com médio porte em altura. O solo predominante da área de estudo é do tipo Latossolo (SEPLAN, 2012).

Para a realização dos trabalhos em campo, foram delimitadas 54 unidades amostrais retangulares de $500 \mathrm{~m}^{2}(10 \times 50 \mathrm{~m})$, totalizando uma área total de 2,7 ha. O tamanho das parcelas foi definido de acordo Felfili et al. (2005), visando representar a estrutura da vegetação e facilitar o controle da mensuração.

Nas parcelas delimitadas foram realizados os seguintes procedimentos de inventários: espécies, diâmetro à altura do peito (DAP) nos indivíduos com diâmetro igual ou superior a 5,0 cm e suas respectivas alturas totais. Na determinação do DAP, nos indivíduos que apresentavam uma ou mais bifurcações, foi utilizado o diâmetro médio quadrático (mediadas a $1,30 \mathrm{~m}$ ) do indivíduo. Para a obtenção das medidas do DAP e da altura total foram utilizados a fita métrica e o hipsômetro Suunto, respectivamente. 
Na estimativa do volume de madeira foi usada a equação de regressão desenvolvida por Berkhout, utilizada no estudo de Imaña-Encinas et al. (2009), em virtude da estrutura da vegetação da área serem semelhantes às do presente estudo. Os critérios estatísticos da equação que basearam a escolha foram o coeficiente de determinação ajustado $\left(\mathrm{R}^{2}{ }_{\mathrm{aj}}\right)$ e o erro padrão da estimava em percentagem (Syx $\left.-\%\right)$ cujo os respectivos valores foram de 0,94 e 15,66:

$$
v=0,02370 * D A P^{2,36540}
$$

em que: DAP é o diâmetro à altura do peito $(\mathrm{m})$ e v é o volume de madeira $\left(\mathrm{m}^{3} \cdot \mathrm{ha}^{-1}\right)$.

Os dados de sensoriamento remoto foram obtidos de uma imagem do sensor OLI Landsat 8 , correspondente ao dia 08/06/2015, data próxima a do levantamento de campo, com órbita/ponto 222/068. Em seguida foi feita a correção atmosférica da imagem para eliminar a interferência atmosférica e poder gerar os valores de reflectância, segundo método descrito por Gürtler et al. (2005), para cada banda do sensor (B2 = azul, B3 = verde, B4 = vermelho, B5 = infravermelho próximo e B7 = infravermelho médio).

Posteriormente, foram calculados dois índices de vegetação (IV), considerados como variáveis adicionais nas análises. Os índices utilizados foram NDVI (Índice de Vegetação Diferença Normalizada) e SAVI (Índice de Vegetação Ajustado para Solo):

$$
N D V I=\frac{B 5-B 4}{B 5+B 4} \quad S A V I=\frac{(1+L)(B 5-B 4)}{B 5+B 4+L}
$$

em que: $B 4$ é a região espectral do vermelho, $B 5$ é a região espectral do infravermelho próximo e L é a constante relacionada a densidade da vegetação.

Os sensores OLI e TM/ETM+ possuem diferenças na ordenação da bandas. Por exemplo, as bandas espectrais utilizadas para a obtenção dos IVs, vermelho e infravermelho próximo, no caso do sensor OLI Landsat 8 correspondem as bandas B4 e B5 respectivamente e nos sensores TM e ETM+, correspondem as bandas B3 e B4,respectivamente.

Os dados de refectância e dos IVs extraídos foram tabulados e sequencialmente procedeu-se as análises estatísticas de correlação e de regressão, em que nesta última foi utilizando o método de Backward, considerando as variáveis do inventário florestal como dependentes segundo o modelo citado por Canavesi et al. (2010):

$$
Y=\beta_{0}+\beta_{1} X_{1}+\beta_{2} X_{2}+\beta_{3} X_{3}+\cdots+\beta_{n} X_{n}+\varepsilon
$$

em que: $\mathrm{Y}$ a variável dependente, $\mathrm{X}$ as variáveis independentes e $\beta$ os coeficientes gerados na seleção das variáveis.

Para a análise dos resíduos foram utilizados os resíduos padronizados, os quais possuem média igual a zero e variância aproximadamente igual a um, com os valores fora do intervalo de -3 e 3 considerados outliers. As melhores equações foram escolhidas através de análise gráfica dos resíduos padronizados, dos menores valores do erro padrão da estimativa absoluto e em porcentagem (Syx e Syx\%) e os maiores valores do coeficiente de determinação ajustado $\left(\mathrm{R}^{2}{ }_{\mathrm{aj}}\right)$, as quais foram utilizadas para a espacialização dos parâmetros florestais para gerar novas imagens com as estimativas desses parâmetros.

\section{RESULTADOS E DISCUSSÃO}

\section{Inventário florestal}

Os dados referentes aos valores máximos, médios e mínimos da coleta de campo são apresentados na tabela 1. A análise dos valores de diâmetro à altura do peito (DAP, $\mathrm{cm})$, área basal $\left(\mathrm{G}, \mathrm{m}^{2} \cdot \mathrm{ha}^{-1}\right)$, altura total $(\mathrm{Ht}$, $\mathrm{m})$, número de árvores $\left(\mathrm{N}\right.$, arv.ha $\left.{ }^{-1}\right)$ e volume $\left(\mathrm{V}, \mathrm{m}^{3} \cdot \mathrm{ha}^{-1}\right)$ obtidos apresentaram médias, respectivamente, de: 11,$42 ; 16,85 ; 6,60 ; 964,91$ e 74,40 .

\begin{tabular}{|c|c|c|c|c|c|}
\hline & DAP (cm) & $\mathbf{G}\left(\mathrm{m}^{2} \cdot \mathrm{ha}^{-1}\right)$ & Ht (m) & $\mathbf{N}\left(\right.$ arv.ha $\left.^{-1}\right)$ & $\mathrm{V}\left(\mathrm{m}^{3} \cdot \mathrm{ha}^{-1}\right)$ \\
\hline Máximo & 20,81 & 36,57 & 9,79 & 2060,00 & 224,23 \\
\hline Média & 11,42 & 16,85 & 6,60 & 964,91 & 74,40 \\
\hline Mínimo & 6,69 & 2,64 & 3,91 & 380,00 & 13,46 \\
\hline CV (\%) & 25,0 & 52,0 & 27,1 & 40,7 & 60,6 \\
\hline
\end{tabular}

Tabela 1. Características dendrométricas do remanescente de Cerrado stricto sensu.

Table 1. Dendrometric caracteristics of the remaining of Cerrado stricto sensu.

Legenda: DAP $(\mathrm{cm})$, diâmetro à altura do peito em centímetros; $\mathrm{G}\left(\mathrm{m}^{2} . \mathrm{ha}^{-1}\right)$, área basal em metros quadrados por hectare; Ht ( $\left.\mathrm{m}\right)$, altura total em metros; $\mathrm{N}\left(\operatorname{arv} \cdot \mathrm{ha}^{-1}\right)$, número de árvores por hectare; $\mathrm{V}\left(\mathrm{m}^{3} \cdot \mathrm{ha}^{-1}\right)$, volume em metros cúbicos por hectare.

FLORESTA, Curitiba, PR, v. 47, n. 1, p. 75 - 83, jan. / mar. 2017.

Santos, M. M. et al.

ISSN eletrônico 1982-4688

DOI: $10.5380 /$ rf.v47i1.47988 


\section{Imagem multiespectral}

Os valores médios de reflectância encontrados das bandas B2, B3, B4, B5 e B7 e os valores dos IVs correspondentes às 54 unidades amostrais são apresentados na tabela 2. Os valores de reflectância da banda B2 (azul) foram muito baixos, com valor médio de 0,00009, enquanto que nas bandas B3 (verde), B4 (vermelho), B5 (infravermelho próximo) e B7 (infravermelho médio) obtiveram-se médias de 0,01732; 0,01329; 0,35053 e 0,02459 , respectivamente.

Estes valores possuem semelhança com os extraídos por Berra et al. (2012) que trabalharam com imagens do sensor TM Landsat 5, em área de plantios de Eucalyptus sp. no Estado de Rio Grande do Sul. Reis et al. (2015) que trabalharam com estratificação em área de Cerrado no Estado de Minas Gerais, com uso de imagens do sensor TM Landsat 5, obtiveram valores médios de reflectância, também, próximos aos obtidos no presente trabalho.

Tabela 2. Médias dos valores de reflectância do Landsat 8 OLI e valores de NDVI e SAVI, das 54 unidades amostrais da área de estudo no município de Cariri do Tocantins.

Table 2. Means of Landsat 8 OLI reflectance values and the NDVI and SAVI values of 54 sampling units of the study area in the Cariri of Tocantins municipality.

\begin{tabular}{lccccccc}
\hline Unidades & \multicolumn{3}{c}{ Reflectância } & \multicolumn{2}{c}{ Índices de vegetação } \\
\cline { 2 - 8 } amostrais & B2 & B3 & B4 & B5 & B7 & NDVI & SAVI \\
\hline Média & 0,00009 & 0,01732 & 0,01329 & 0,35053 & 0,02459 & 0,92170 & 0,57786 \\
\hline
\end{tabular}

Legenda: B2, B3, B4, B5 e B7 referem-se a reflectância das bandas na região do azul, verde, vermelho, infravermelho próximo e SWIR 2 respectivamente; NDVI, índice de vegetação por diferença normalizada; SAVI, índice de vegetação ajustado para o solo.

\section{Análise dos dados}

Em primeiro lugar são apresentados os valores de correlação entre as variáveis independentes (reflectância das diferentes bandas e os índices de vegetação) e as variáveis dependentes (parâmetros dendrométricos) os quais são obtidos através do emprego de função linear, visando determinar o grau de associação entre as variáveis (Tabela 3).

Para a variável dependente DAP, as maiores correlações foram encontradas entre as variáveis independentes $\operatorname{NDVI}^{3}(-0,47)$, B4 e B7, ambas com mesmo valor $(0,46)$. Para a variável $\mathrm{G}$, os destaques continuaram sendo entre a variável $\mathrm{NDVI}^{3}(0,77)$ e a variável da banda espectral B4 $(0,76)$. Para $\mathrm{Ht}$, as correlações não foram significativas tendo como maiores coeficientes as variáveis $\mathrm{B} 3$ e B5 $5^{3}$, ambas com 0,25 . Assim como em $\mathrm{Ht}$, para $\mathrm{N}$ as correlações não foram significativas obtendo-se como maiores coeficientes entre $\mathrm{B} 4, \mathrm{NDVI}^{2} \mathrm{e}$ $\mathrm{NDVI}^{3}$, todas com valor de 0,17 . Para $\mathrm{V}$, a variável $\mathrm{NDVI}^{3}$ continuou sendo destaque entre as correlações $(-0,61)$ juntamente com a B4 $(0,60)$. Com exceção para a variável dependente $\mathrm{Ht}$, a variável independente $\mathrm{NDVI}^{3}$ obteve maiores correlações entre as outras quatro variáveis dependentes do estudo. $\mathrm{O}$ fato de alguns coeficientes terem valores negativos, aponta somente que algumas variáveis possuem correlações inversas.

Reis et al. (2015) apresentaram em seu trabalho melhores correlações entre volume e a banda do infravermelho próximo $(-0,638)$ e infravermelho médio $(-0,501)$, sendo que a correlação com o NDVI foi de 0,187 , o que não diferiu muito em relação ao observado na tabela 3. Berra et al. (2012) encontraram maiores valores de correlação da reflectância da banda do vermelho $(-0,78)$ e dos índices de vegetação NDVI $(0,79)$ e SR $(0,82)$ com o volume $\left(\mathrm{m}^{3} \cdot \mathrm{ha}^{-1}\right)$. Lu et al. (2004), chegaram a correlações com um certo grau de similaridade em relação aos parâmetros avaliados no presente trabalho.

Os parâmetros G, DAP e $\mathrm{Ht}$ (comuns em ambos os trabalhos), obtidos por Lu et al. (2004), tiveram maiores correlações entre as variáveis das bandas do infravermelho médio $(0,58)$ e infravermelho próximo $(0,51)$, infravermelho médio $(0,79)$ e banda do vermelho $(0,68)$ e infravermelho médio $(0,85)$ e banda do verde $(0,83)$, para cada parâmetro respectivamente.

Ponzoni e Shimabukuro (1998), avaliando a relação existente entre os índices de vegetação NDVI e SAVI e parâmetros dendrométricos em plantios de Eucalyptus sp., utilizando imagens do sensor Landsat 5 TM, encontraram correlações entre NDVI e os parâmetros dendrométricos volume, área basal, altura e número de árvores, de 0,$33 ; 0,37 ; 0,21$ e $-0,12$, respectivamente. Entre SAVI e os mesmos parâmetros citados anteriormente, de 0,$33 ; 0,41 ; 0,16 \mathrm{e}-0,03$, respectivamente.

Watzlawick et al. (2009), apesar de trabalhar com o satélite IKONOS II e ter como variáveis dependentes desejadas biomassa aérea e carbono orgânico, encontrou maior correlação entre os valores de reflectância da região do infravermelho próximo, os índices de vegetação: Razão, NDVI e SAVI.

Resultados estes que contrariam aos do presente estudo, pois as maiores correlações se encontraram na maioria dos casos com a banda do vermelho (B4) e não com a banda do infravermelho próximo (B5), que apresentou baixos valores de correlação. Segundo os autores supracitados, as bandas B4 e B5 apresentam respectivamente regiões de forte absorção e máxima reflectância do espectro eletromagnético. 
Tabela 3. Coeficientes de correlação de Pearson entre as variáveis dendrométricas obtidas em campo (dependentes) e as variáveis obtidas da imagem Landsat 8 OLI (independentes) da área de estudo.

Table 3. Pearson correlation coefficient between the dendrometric variables obtained in field (dependent) and the variables obtained of Landsat 8 OLI image (independent) of study area.

\begin{tabular}{|c|c|c|c|c|c|}
\hline Variável & DAP & $\mathbf{G}$ & Ht & $\mathbf{N}$ & $\mathbf{V}$ \\
\hline B2 & $0,2707^{*}$ & $0,2840^{*}$ & $-0,0108$ & $-0,0451$ & 0,2440 \\
\hline B3 & $0,4175^{* *}$ & $0,6224^{* *}$ & 0,2490 & 0,1364 & $0,4899^{* *}$ \\
\hline B4 & $0,4651^{* *}$ & $0,7626^{* *}$ & 0,1507 & 0,1700 & $0,6007^{* *}$ \\
\hline B5 & $-9,6 E-5$ & $-0,1979$ & 0,2331 & $-0,0862$ & $-0,1565$ \\
\hline B7 & $0,4650^{* *}$ & $0,6904^{* *}$ & 0,1568 & 0,1367 & $0,5474^{* *}$ \\
\hline NDVI & $-0,4494^{* * *}$ & $-0,7388^{* * *}$ & $-0,1560$ & $-0,1685$ & $-0,5813^{* *}$ \\
\hline SAVI & $-0,1811$ & $-0,4273^{* *}$ & 0,1250 & $-0,1021$ & $-0,3355^{*}$ \\
\hline $\mathrm{B}^{2}$ & $0,2931^{*}$ & 0,2519 & $-0,0373$ & $-0,0653$ & 0,2559 \\
\hline $\mathbf{B 3}^{2}$ & $0,4225^{* *}$ & $0,6609^{* *}$ & 0,2081 & 0,1153 & $0,5172^{* *}$ \\
\hline$B 4^{2}$ & $0,3813^{* *}$ & $0,6558^{* *}$ & 0,1550 & 0,1418 & $0,4971^{* *}$ \\
\hline $\mathrm{B5}^{2}$ & 0,0232 & $-0,2009$ & 0,2424 & $-0,1277$ & $-0,1605$ \\
\hline$B 7^{2}$ & $0,4454^{* *}$ & $0,7194^{* *}$ & 0,1627 & 0,1604 & $0,5541^{* *}$ \\
\hline $\mathrm{NDVI}^{2}$ & $-0,4637^{* *}$ & $-0,7542^{* *}$ & $-0,1534$ & $-0,1716$ & $-0,5988^{* *}$ \\
\hline SAVI $^{2}$ & $-0,1480$ & $-0,4001^{* *}$ & 0,1573 & $-0,1155$ & $-0,3177^{*}$ \\
\hline $\mathbf{B} 2^{3}$ & $0,2976^{*}$ & 0,2404 & $-0,0449$ & $-0,0708$ & 0,2575 \\
\hline $\mathbf{B 3}^{3}$ & $0,3989^{* * *}$ & $0,6509^{* *}$ & 0,1685 & 0,1067 & $0,5038^{* *}$ \\
\hline$B 4^{3}$ & $0,2973^{*}$ & $0,5511^{* *}$ & 0,1441 & 0,1199 & $0,3894^{* *}$ \\
\hline $\mathrm{B5}^{3}$ & 0,0485 & $-0,1983$ & 0,2498 & $-0,1656$ & $-0,1581$ \\
\hline$B 7^{3}$ & $0,4036^{* *}$ & $0,7028^{* * *}$ & 0,1598 & 0,1680 & $0,5239^{* *}$ \\
\hline $\mathrm{NDVI}^{3}$ & $-0,4742^{* *}$ & $-0,7650^{* *}$ & $-0,1501$ & $-0,1742$ & $-0,6116^{* *}$ \\
\hline SAVI $^{3}$ & $-0,1123$ & $-0,3710^{* *}$ & 0,1837 & $-0,1311$ & $-0,2960^{*}$ \\
\hline LOG B5 & $-0,0190$ & $-0,1890$ & 0,2221 & $-0,0442$ & $-0,1455$ \\
\hline LOG B7 & $0,4473^{* *}$ & $0,5988^{* *}$ & 0,1324 & 0,0908 & $0,4888^{* * *}$ \\
\hline LOG NDVI & $-0,4312^{* *}$ & $-0,7186^{* *}$ & $-0,1577$ & $-0,1647$ & $-0,5589^{* *}$ \\
\hline LOG SAVI & $-0,2082$ & $-0,4495^{* *}$ & 0,0877 & $-0,0922$ & $-0,3467^{*}$ \\
\hline
\end{tabular}

Legenda: B2, B3, B4, B5 e B7, reflectância das bandas do azul, verde, vermelho, infravermelho próximo e SWIR 2, respectivamente; NDVI, índice de vegetação por diferença normalizada; SAVI, índice de vegetação ajustado para o solo; $\mathrm{B}^{2}, \mathrm{~B}^{2}, \mathrm{~B}^{2}, \mathrm{~B}^{2}, \mathrm{~B}^{2}, \mathrm{NDVI}^{2}$ e $\mathrm{SAVI}{ }^{2}$, reflectância das bandas e índices de vegetação elevado ao quadrado; $3^{3}$, B3 ${ }^{3}, \mathrm{~B}^{3}{ }^{3}, \mathrm{~B}^{3}{ }^{3}, \mathrm{~B}^{3}{ }^{3}, \mathrm{NDVI}^{3}$ e $\mathrm{SAVI}^{3}$, reflectância das bandas e índices de vegetação elevado ao cubo; LOG B5, LOG B7, LOG NDVI e LOG SAVI, logaritmo na base 10 das bandas B5, B7, NDVI e SAVI respectivamente; $* *$ significativo a $1 \%$ e $*$ significativo a $5 \%$ pelo teste t.

Os melhores modelos gerados para cada variável dependente, a partir dos dados de reflectância e IVs da imagem, através de regressão linear pelo método de Backward são apresentados na tabela 4. Foram escolhidos dois modelos de cada parâmetro que melhor representaram com base nas análises estatísticas empregadas (F, $\mathrm{R}_{\mathrm{aj}}{ }_{\mathrm{aj}}$, Syx e Syx em porcentagem).

Foram obtidas duas equações de regressão para cada variável dependente, sendo as equações de números ímpares $(1,3,5,7$ e 9) ajustadas com as variáveis independentes elevadas à segunda potência e as equações pares $(2,4,6,8$ e 10) ajustadas com todas as variáveis independentes do estudo.

Os valores de $\mathrm{R}^{2}$ ajustados $\left(\mathrm{R}_{\mathrm{aj}}{ }\right)$ para DAP, G, Ht, $\mathrm{N}$ e V variaram de 0,57 a 0,58, de 0,61 a 0,66, de 0,015 a 0,16 , de 0,25 a 0,34 e de 0,40 a 0,49 , respectivamente. Berra et al. (2012), trabalhando somente com estimativa de volume, tiveram como $\mathrm{R}_{\text {aj }}$ variando de 0,61 a 0,68. Canavesi et al. (2010), com o objetivo de quantificar volume de madeira e o impacto do sombreamento do relevo com a aplicação de dados hiperespectrais, obtiveram valores de $\mathrm{R}^{2}$ aj variando de 0,59 a 0,70 utilizando o método de stepwise.

Watzlawick et al. (2009) utilizando o mesmo método de seleção, porém com a finalidade de quantificar biomassa arbórea, encontraram valores de $\mathrm{R}^{2}$ aj variando desde 0,02 até 0,73 . Barros et al. (2015), quantificando volume, obtiveram equações com $\mathrm{R}^{2}$ aj variando de 0,13 a 0,38. Lima Júnior et al. (2014), utilizando equações alométricas para a quantificação de biomassa a partir de índice de vegetação, encontraram valores de $\mathrm{R}^{\mathbf{2}}$ aj $\mathrm{de} 0,70$.

Na figura 1 é demonstrado graficamente a dispersão dos resíduos dos modelos que levaram todas as variáveis independentes $(2,4,6,8$ e 10) apresentados na tabela 4, pois os mesmos obtiveram melhores análises. Nota-se que a distribuição de resíduos padronizados para as variáveis DAP, G e V, encontram-se mais próximos da linha média de distribuição, demonstrando que os valores estimados pelas equações geradas para esses parâmetros, explicaram melhor a relação valor real com valor estimado. 
Tabela 4. Modelos de regressão linear testados e ajustados para estimar as variáveis dependentes.

Table 4. Linear regression models tested and adjusted to estimate the dependent variables.

\begin{tabular}{|c|c|c|c|c|c|c|}
\hline $\mathbf{N}^{\circ}$ & $\begin{array}{l}\text { Variável } \\
\text { dependente }\end{array}$ & Modelo de regressão & F & $\mathbf{R}^{2}{ }_{\text {aj }}$ & Syx & $\begin{array}{l}\text { Syx } \\
(\%)\end{array}$ \\
\hline 1 & DAP & $\begin{array}{l}-66,28+\mathrm{B} 2 \cdot(2 \cdot 307,98)+\mathrm{B} 3 \cdot(-318,16)+\mathrm{B} 7 \cdot(367,14)+\mathrm{NDVI} \cdot(251,76)+ \\
\mathrm{B}^{2} \cdot(153,92)+\mathrm{B}^{2} .(-11 \cdot 256,20)+\mathrm{NDVI}^{2} .(-171,09)+\mathrm{SAVI}^{2} .(-68,23)+\varepsilon\end{array}$ & 7,56 & 0,50 & 2,02 & 17,65 \\
\hline 2 & DAP & 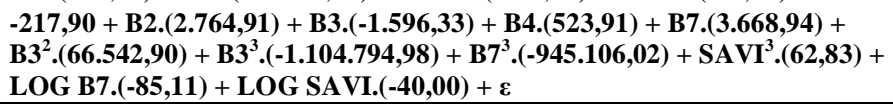 & $\mathbf{7 , 8 0}$ & 0,57 & 1,88 & 16,47 \\
\hline 3 & G & $\begin{array}{l}-198,11+\mathrm{B} 3 \cdot(-723,29)+\mathrm{B} 4 \cdot(1.423,68)+\mathrm{NDVI} \cdot(395,48)+\mathrm{B} 5^{2} \cdot(83,54)+ \\
\mathrm{NDVI}^{2} \cdot(-194,17)+\varepsilon\end{array}$ & 18,57 & 0,63 & 5,34 & 31,68 \\
\hline 4 & G & $\begin{array}{l}0,92+\text { B4. }(1.475,09)+\text { B3 }^{2} .(-72.262,25)+B^{3} .(1.707 .213,96)+\mathbf{B}^{3} .(- \\
250.265,24)+ \text { SAVI }^{3} .(55,40)+\varepsilon\end{array}$ & 20,50 & 0,66 & 5,16 & 30,64 \\
\hline 5 & $\mathrm{Ht}$ & $37,23+\mathrm{B} 4 \cdot(-204,64)+\mathrm{B}^{2} \cdot(-3 \cdot 657,78)+\mathrm{NDVI}^{2} \cdot(-33,98)+\mathrm{SAVI}^{2} .(10,84)+\varepsilon$ & 2,93 & 0,13 & 1,67 & 25,31 \\
\hline 6 & $\mathrm{Ht}$ & $\begin{array}{l}1,39+\mathrm{B2}^{3} \cdot(-38.189 .521,43)+\mathrm{NDVI}^{3} \cdot(-9,11)+\mathrm{SAVI}^{3} \cdot(11,90)+\text { LOG B7.(- } \\
6,17)+\varepsilon\end{array}$ & $\mathbf{3 , 4 4}$ & 0,16 & 1,64 & 24,89 \\
\hline 7 & $\mathrm{~N}$ & $\begin{array}{l}-1.329,92+\text { B2.(-296.088,26)+B3.(17.515,13) + B42 } .(729.450,64)+B 5^{2} .(- \\
26.058,13)+ \text { SAVI }^{2} .(14.826,84)+\varepsilon\end{array}$ & 3,45 & 0,19 & 353,59 & 36,65 \\
\hline 8 & $\mathrm{~N}$ & $\begin{array}{l}-4.692,72+\text { B2. }(-1.411 .441,89)+\text { B3. }(209.959,60)+\text { B3 }^{2} \cdot(13.842 .256,31)+ \\
\text { B2 }^{3} .(55.337 .011 .502,71)+\text { B3 }^{3} .(266.067 .746,65)+\text { B4 }^{3} .(-28.856,75)+ \\
\text { B7 }^{3} .(24.338 .256,88)+\text { SAVI }^{3} .(9.349,08)+\text { LOG B7. }(-2.440,42)+\varepsilon\end{array}$ & 3,96 & $\mathbf{0 , 3 4}$ & 319,63 & 33,13 \\
\hline 9 & $\mathrm{~V}$ & $\begin{array}{l}-3.202,73+\mathrm{B} 2 .(-162.330,33)+\mathrm{B} 3 .(-3.546,19)+\mathrm{NDVI} .(7.269,69)+ \\
\mathrm{B}^{2} .(42.548 .490,43)+\mathrm{B}^{2} .(171 \cdot 926,16)+\mathrm{NDVI}^{2} .(-4.099,87)+ \\
\mathrm{SAVI}^{2} .(273,62)+\varepsilon\end{array}$ & 7,65 & 0,47 & 32,75 & 44,01 \\
\hline 10 & V & $\begin{array}{l}-2.216,68+\text { B2. }(22.959,72)+\text { B3. }(-3.412,95)+\text { B7. }(35.277,02)+ \\
\text { B4 }^{2} .(273.194,26)+\text { B4 } 4^{3} .(-3.577 .958,31)+B 7^{3}(-8.159 .551,90)+ \\
\text { SAVI }{ }^{3} .(220,95)+\text { LOG B7.(-941,36) }+\varepsilon\end{array}$ & 7,12 & 0,49 & 32,35 & 43,48 \\
\hline
\end{tabular}

Legenda: $\mathrm{N}^{\circ}$, número do modelo; DAP, diâmetro a altura do peito $(\mathrm{cm})$; $\mathrm{G}$, área basal $\left(\mathrm{m}^{2} / \mathrm{ha}\right)$; Ht, altura total (m); $\mathrm{N}$, número de árvores $\left(\right.$ arv.ha $\left.^{-1}\right) ; \mathrm{V}$, volume de madeira $\left(\mathrm{m}^{3} \cdot \mathrm{ha}^{-1}\right) ; \mathrm{F}$, valor de $\mathrm{F}$ da análise de variância; $\mathrm{R}_{\mathrm{aj}}^{2}$, coeficiente de determinação ajustado; Syx, erro padrão da estimativa; $\operatorname{Syx}(\%)$, erro padrão da estimativa em porcentagem e $+\varepsilon$, erro do modelo.
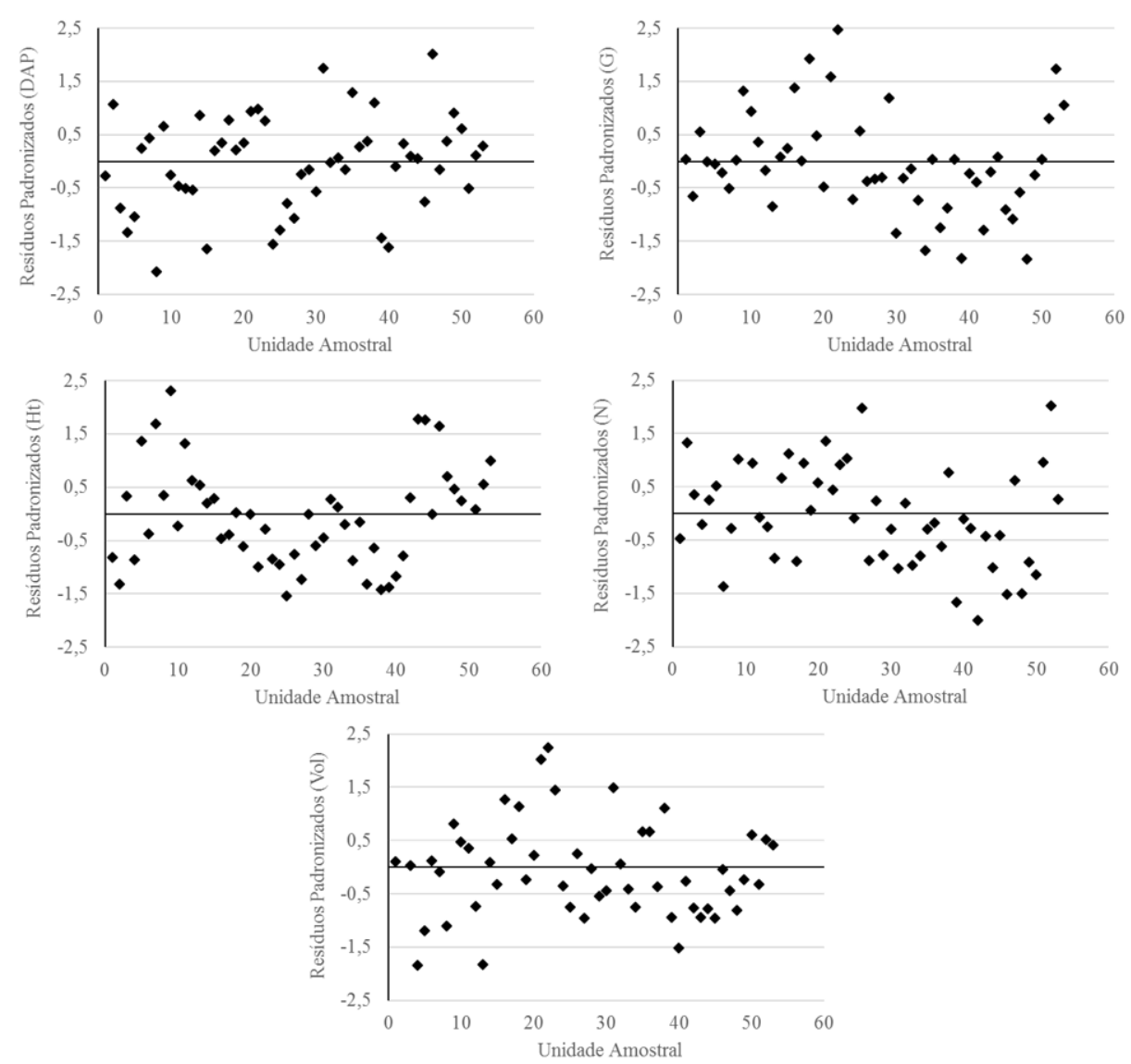

Figura 1. Distribuição dos resíduos padronizados dos modelos selecionados para estimativa dos parâmetros florestais avaliados.

Figure 1. Distribution of the standardized residuals of the selected models for estimating forest parameters studied. 


\section{Espacialização}

A espacialização foi realizada com os melhores modelos de regressão, um de cada variável, de acordo com os parâmetros de análise utilizados no estudo, onde foram geradas novas imagens para cada variável dependente, que estão representados na figura 2.

Nota-se que na região com descontinuação de vegetação houve boa representação dos produtos em relação aos observados na imagem original, com baixos valores em todos os parâmetros analisados (DAP, G, Ht, $\mathrm{N}$ e V). Assim, também, acontece com a vegetação de mata de galeria, que é caracterizada pelos baixos valores de DAP, $\mathrm{Ht}$ de médio porte, $\mathrm{G}$ e $\mathrm{V}$ de baixos valores e $\mathrm{N}$ razoável. Para $\mathrm{Ht}$ e $\mathrm{N}$, mesmo com baixos valores de $\mathrm{R}^{2}{ }_{\mathrm{aj}}$ é possível observar boa representação (espacialização) em toda a área de estudo, comprovando a boa eficiência da metodologia.
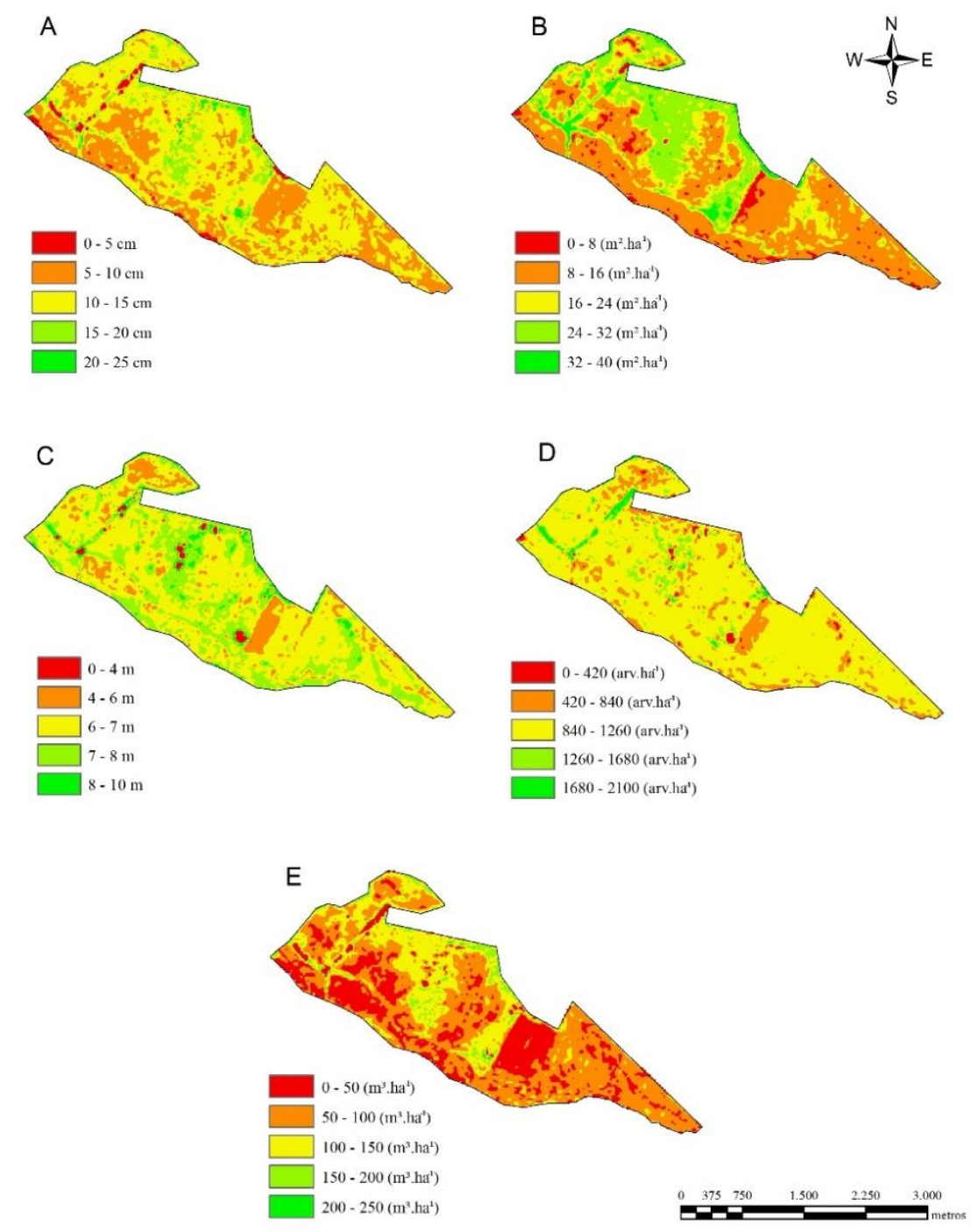

Figura 2. Espacialização dos resultados com os melhores modelos para cada variável: A, diâmetro a altura do peito $(\mathrm{cm}) ; \mathrm{B}$, área basal $\left(\mathrm{m}^{2} \cdot \mathrm{ha}^{-1}\right) ; \mathrm{C}$, altura total $(\mathrm{m}) ; \mathrm{D}$, número de árvores $\left(\operatorname{arv}^{-1} \mathrm{~h}^{-1}\right)$ e E, volume de madeira $\left(\mathrm{m}^{3} \cdot \mathrm{ha}^{-1}\right)$.

Figure 2. Spatialization of results with the better models to each variable: A, diameter at breast height $(\mathrm{cm}) ; \mathrm{B}$, basal area $\left(\mathrm{m}^{2} \cdot \mathrm{ha}^{-1}\right) ; \mathrm{C}$, total height $(\mathrm{m})$; D, number of trees $\left(\operatorname{arv} \cdot \mathrm{ha}^{-1}\right)$ and $\mathrm{E}$, timber volume $\left(\mathrm{m}^{3} \cdot \mathrm{ha}^{-1}\right)$.

\section{CONCLUSÕES}

Com os resultados obtidos no presente trabalho, pode-se concluir que:

- As estimativas dos parâmetros florestais analisados através de análise de regressão, utilizando imagens do sensor OLI Landsat 8, apresentaram melhores resultados estatísticos para os parâmetros DAP, G e V,

FLORESTA, Curitiba, PR, v. 47, n. 1, p. 75 - 83, jan. / mar. 2017.

Santos, M. M. et al.

ISSN eletrônico 1982-4688

DOI: $10.5380 /$ rf.v47i1.47988 
demonstrando o potencial de utilização de tal metodologia em áreas extensas, remotas e de difícil caracterização como são as áreas de bioma cerrado.

- Dentre os índices de vegetação, o NDVI apresentou melhores correlações com os parâmetros estudados, sendo em todas as situações tidas como correlações inversas.

- Dentre as bandas espectrais do sensor OLI Landsat 8, apresentam destaque as bandas B3 (verde), B4 (vermelho) e B7 (infravermelho médio), com melhores correlações com os parâmetros florestais.

- As equações ajustadas com todas as variáveis independentes (2, 4, 6, 8 e 10) apresentaram os melhores resultados estatísticos.

\section{AGRADECIMENTOS}

A CAPES pela concessão de bolsa de pós-doutorado no país ao terceiro autor (processo n ${ }^{\circ} 88887.116024 / 2016$ $00)$.

\section{REFERÊNCIAS}

ALMEIDA, A. Q. de; RIBEIRO, A.; DELGADO, R. C.; RODY, Y. P.; OLIVEIRA, A. S. de; LEITE, F. P. Índice de área foliar de Eucalyptus estimado por índices de vegetação utilizando imagens TM-Landsat 5. Floresta e Ambiente, v. 22, n. 3, p. 368-376, 2015.

ALVES, M. V. G.; CHIAVETTA, U.; KOEHLER, H. S.; MACHADO, S. do A.; KIRCHNER, F. F. Aplicação de k-nearest neighbor em imagens multispectrais para a estimativa de parâmetros florestais. Floresta, Curitiba, V. 43, n. 3, p. 351-362, 2013.

BARros, B. S. X. de; GUERRA, S. P. S.; BARROS, Z. X. de; CATITA, C. M. S.; FERNANDES, J. C. C. C. Uso de imagens de satélite para cálculo de volume em floresta de eucalipto no município de Botucatu/SP. Energia na Agricultura, Botucatu, v. 30, n. 1, p. 60-67, 2015.

BERRA, E. F.; BRANDELERO, C.; PEREIRA, R. S.; SEBEM, E.; GOERGEN, L. C. de G.; BENEDETTI, A. C. P.; LIPPERT, D. B. Estimativa do volume total de madeira em espécies de eucalipto a partir de imagens de satélite Landsat. Ciência Florestal, Santa Maria, v. 22, n. 4, p. 853-864, 2012.

BOLFE, E. L.; PEREIRA, R. S.; MADRUGA, P. R. de A. Geoprocessamento e sensoriamento remoto aplicados à análise de recursos florestais. Ciência Rural, Santa Maria, v. 34, n. 1, p. 105-111, 2004.

CANAVESI, V.; PONZONI, F. J.; VALERIANO, M. M. Estimativa de volume de madeira em plantios de Eucalyptus spp. utilizando dados hiperespectrais e dados topográficos. Revista Árvore, Viçosa, v. 34, n. 3, p. 539-549, 2010.

CASTANHEIRA, L. B.; LANDIM, P. M. B.; LOURENÇO, R. W. Variabilidade do índice de vegetação por diferença normalizada (NDVI) em áreas de reflorestamento: Floresta Estadual 'Edmundo Navarro de Andrade' (FEENA)/Rio Claro (SP). Geociências, São Paulo, v. 33, n. 3, p. 449-456, 2014.

FERRAZ, A. S.; SOARES, V. P.; SOARES, C. P. B.; RIBEIRO, C. A. A. S.; BINOTI, D. H. B.; LEITE, H. G. Estimativa do estoque de biomassa em um fragmento florestal usando imagens orbitais. Floresta e Ambiente, Seropédica, v. 21, n. 3, p. 286-296, 2014.

GÜRTLER, S.; EPIPHANIO, J. C. N.; LUIZ, A. J. B.; FORMAGGIO, A. R. Planilha eletrônica para o cálculo da reflectância em imagens TM e ETM+ Landsat. Revista Brasileira de Cartografia, Rio de Janeiro, n. 57/02, p. 162-167, 2005.

HENTZ, A. M. K.; RUZA, M. S.; CORTE, A. P. D.; SANQUETTA, C. R. Técnicas de sensoriamento remoto para estimativa de biomassa em ambientes florestais. Enciclopédia Biosfera, Goiânia, v. 10, n. 18; p. 28102823, 2014.

HUETE, A. R. Soil influences in remotely sensed vegetation canopy spectra. In: ASRAR, G. (ed.). Theory and applications of optical remote sensing. New York: John Wiley \& Sons, 1989, p. 107-141.

IMAÑA-ENCINAS, J.; SANTAN, O. A.; PAULA, J. E. de; IMAÑA, C. R. Equações de volumen de madeira para o cerrado de Planaltina de Goiás. Floresta, Curitiba, v. 39, n. 1, p. 107-116, 2009. 
LIMA JÚNIOR, C. de.; ACCIOLY, L. J. de O.; GIONGO, V.; LIMA, R. L. F. de A.; SAMPAIO, E. V. de S. B.; MENEZES, R. S. C. Estimativa de biomassa lenhosa da caatinga com uso de equações alométricas e índice de vegetação. Scientia Forestalis, Piracicaba, v. 42, n. 102, p. 289-298, 2014.

LEAL, F. A.; MATRICARDI, E. A. T.; OLIVEIRA, K. A. de; ALMEIDA, G. da S. Índice de vegetação na estimativa do volume em um povoamento de eucalipto. Enciclopédia Biosfera, Goiânia, v. 9, n. 17; p. 16381646, 2013.

LU, D.; MAUSELB, P.; BRONDÍZIO, E.; MORAN, E. Relationships between forest stand parameters and Landsat TM spectral responses in the Brazilian Amazon Basin. Forest Ecology and Management, Amsterdan, v. 198, n. 1-3, p. 149-167. 2004.

PONZONI, F. J.; SHIMABUKURO, Y. E. Relação entre índice de vegetação (NDVI e SAVI) e parâmetros biométricos de plantios de Eucalyptus urophylla e Eucalyptus camaldulensis em Cachoeira do Manteiga-MG. Revista Árvore, Viçosa, v. 22, n. 3, p. 357-366, 1998.

REIS, A. A. dos; MELLO, J. M. de; ACERBI JÚNIOR, F. W.; CARVALHO, L. M. T. de. Estratificação em cerrado sensu stricto a partir de imagens de sensoriamento remoto e técnicas geoestatísticas. Scientia Florestalis, Piracicaba, v. 43, n. 106, p. 377-386. 2015.

RIBEIRO, S. C.; JACOVINE, L. A. G.; SOARES, C. P. B.; MARTINS, S. V.; SOUZA, A. L. de; NARDELLI, A. M. B. Quantificação de biomassa e estimativa de estoque de carbono em uma floresta madura no município de Viçosa, Minas Gerais. Revista Árvore, Viçosa, v. 33, n. 5, p. 917-926, 2009.

SECRETARIA DO PLANEJAMENTO. SUPERINTENDÊNCIA DE PLANEJAMENTO E GESTÃO CENTRAL DE POLÍTICAS PÚBLICAS (SEPLAN). Diretoria de Zoneamento Ecológico-Econômico (DZE). Base de dados geográficos do Tocantins. Palmas, SEPLAN/ DZE, 2012. CD-ROM.

VIANA, H.; ARANHA, J.; LOPES, D.; COHEN, W. B. Estimation of crown biomass of Pinus pinaster stands and shrubland above-ground biomass using forest inventory data, remotely sensed imagery and spatial prediction models. Ecological Modelling, Amsterdan, v. 226, p. 22-35, 2012.

WATZLAWICK, L. F.; KIRCHNER, F. F.; SANQUETTA, C. R. Estimativa de biomassa e carbono em floresta com Araucária utilizando imagens do satélite Ikonos II. Ciência Florestal, Santa Maria, v. 19, n. 2, p. 169-181, 2009. 